\title{
Calcified Middle Cranial Fossa Mass
}

\author{
${ }^{1}$ Department of Neurological Surgery, University of Texas \\ Southwestern Medical Center, Dallas, Texas, United States \\ 2 Department of Pathology, University of Texas Southwestern Medical \\ Center, Dallas, Texas, United States \\ ${ }^{3}$ Department of Otolaryngology, University of Texas Southwestern \\ Medical Center, Dallas, Texas, United States
}

James Botros $^{1}$ Kimmo Hatanpaa ${ }^{2}$ Brandon Isaacson ${ }^{3}$ Samuel L. Barnett ${ }^{1}$

\author{
Address for correspondence Samuel L. Barnett, MD, Department of \\ Neurological Surgery, University of Texas Southwestern Medical \\ Center, 5323 Harry Hines Boulevard, Dallas, TX 75390-8855, \\ United States (e-mail: sam.barnett@utsouthwestern.edu).
}

J Neurol Surg Rep 2017;78:e34-e36.

\begin{abstract}
Keywords

- osteosarcoma

- middle fossa

- neoplasms of connective and soft tissue

A 21-year-old male presented for evaluation of transient loss of consciousness and was found to have a hyperdense mass in the left middle fossa. He underwent craniotomy for tumor resection. Intra- and extradural invasion was noted. Gross total resection was achieved. Pathology demonstrated a densely cellular neoplasm with predominately spindle cell morphology in a collagen-containing stroma, areas of vascular proliferation, focal mineralization, and regions of cartilage formation. High mitotic index and regions of necrosis were seen. Based on the final diagnosis of osteosarcoma, the patient was referred for chemotherapy and radiation. Intracranial osteosarcoma is a nonmeningiomatous mesenchymal tumor. Most osteosarcomas are meningeal-based and supratentorial. Presentation depends on tumor location and may include focal neurologic deficits, cranial neuropathy, seizures, or symptoms of increased intracranial pressure. Given the relative rarity of intracranial osteosarcoma, there are no established guidelines for treatment, and therapy is guided by experience with systemic osteosarcoma. Gross total resection is recommended whenever feasible. Both chemotherapy and radiation therapy are used as adjuvant therapy. Regardless of treatment, osteosarcoma remains a highly aggressive malignancy with a poor prognosis. Morbidity and mortality may be the result of local recurrence or development of pulmonary or skeletal metastasis.
\end{abstract}

\section{Calcified Middle Cranial Fossa Mass}

A 21-year-old male presented for evaluation of transient loss of consciousness. He complained of auricular fullness, diminished hearing, and left facial tingling. He denied diplopia or facial weakness. Examination demonstrated a partial left oculomotor palsy with ptosis and mydriasis. Facial strength and sensation were normal. Hearing was diminished on the left. Motor examination was symmetric. A firm, rubbery mass was palpable superior to the left zygoma. Head computed tomography demonstrated a hyperdense mass in the left middle fossa (-Fig. 1), with erosion of the petrosquamous temporal bone and sphenoid wing. Magnetic reso- nance imaging (MRI) of the brain demonstrated an avidly enhancing mass in the left middle fossa, with mass effect on the left temporal lobe and effacement of the lateral ventricle (-Fig. 2).

He underwent left pterional craniotomy and infratemporal fossa approach for tumor resection with autologous fat graft. The tumor was pink-tan, firm, and lobulated, with numerous calcifications. Intra- and extradural invasion was noted. Gross total resection was achieved (- Fig. 3).

Pathology demonstrated a densely cellular neoplasm with predominately spindle cell morphology in a collagencontaining stroma, focal areas of hemangiopericytoma-like vascular proliferation, and focal mineralization (-Fig. 4). received July 20, 2016 accepted after revision December 21, 2016
DOI http://dx.doi.org/ $10.1055 / \mathrm{s}-0037-1598112$. ISSN 2193-6358. (c) 2017 Georg Thieme Verlag KG Stuttgart · New York
License terms

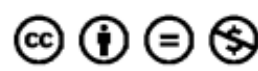




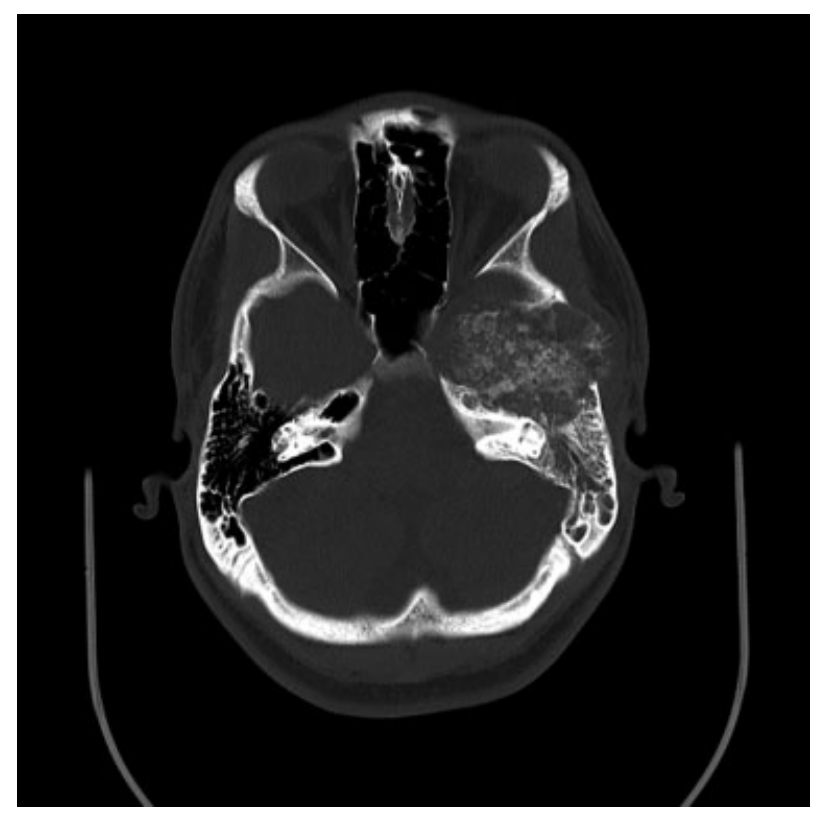

Fig. 1 Computed tomography scan demonstrating hyperdense left middle cranial fossa mass with osteolysis.

Well-defined regions of cartilage formation were noted (-Fig. 5). High mitotic index and regions of necrosis were seen. Immunohistochemistry for S100, epithelial membrane antigen (EMA), glial fibrillary acidic protein (GFAP), and synaptophysin was negative. Based on the final diagnosis of osteosarcoma, the patient was referred for chemotherapy and radiation.

Intracranial osteosarcoma belongs to the class of nonmeningiomatous mesenchymal tumors, which encompass benign lesions, such as lipoma, and more malignant lesions, such as hemangiopericytoma, osteosarcoma, and chondro-

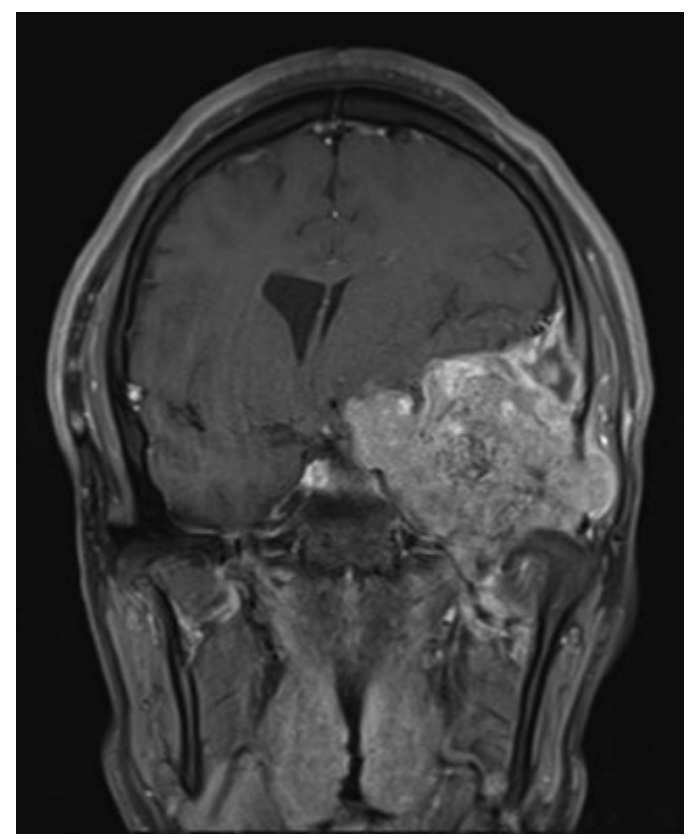

Fig. 2 Magnetic resonance imaging demonstrating left middle fossa mass with avid gadolinium enhancement.

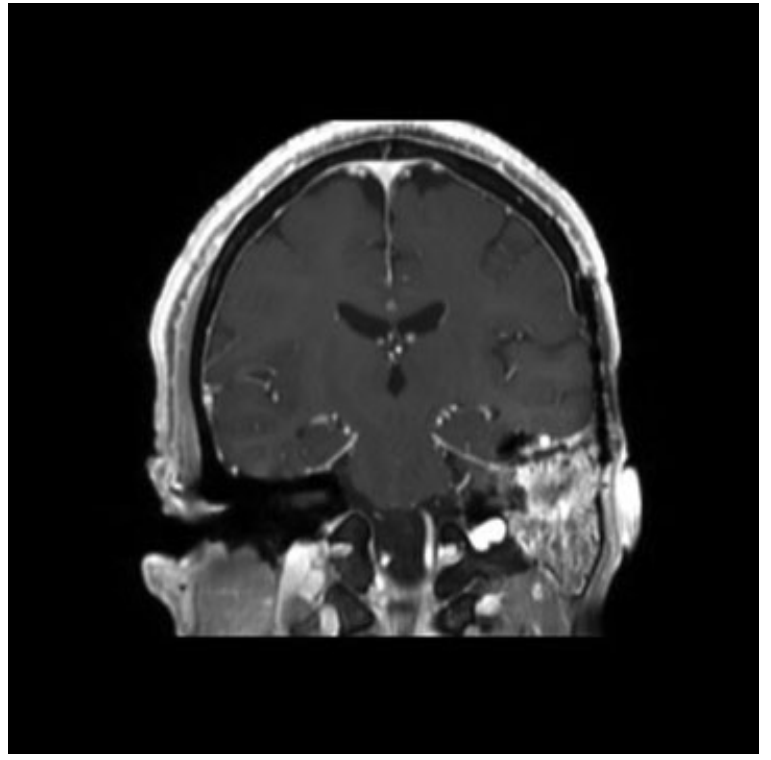

Fig. 3 Postoperative magnetic resonance imaging demonstrating gross total resection of left middle fossa mass.

sarcoma. These tumors are relatively rare, with sarcomas comprising between 0.2 and $2.5 \%$ of intracranial tumors. ${ }^{1}$ In contrast to long bone osteosarcoma, intracranial osteosarcoma is more common in adults. There is no clear gender predilection. Most osteosarcomas are meningeal-based and supratentorial. Presentation depends on tumor location and may include focal neurologic deficits, cranial neuropathy, seizures, or symptoms of increased intracranial pressure.

Radiographic evaluation of osteosarcoma typically demonstrates intralesional calcification. Osteolysis is common. The tumors are typically isointense on T1-weighted MRI and hyperintense on T2-weighted imaging. ${ }^{2}$ MRI may demonstrate areas of hemorrhage or necrosis. Although osteosarcoma may appear well-circumscribed on imaging, there is typically some degree of parenchymal infiltration.

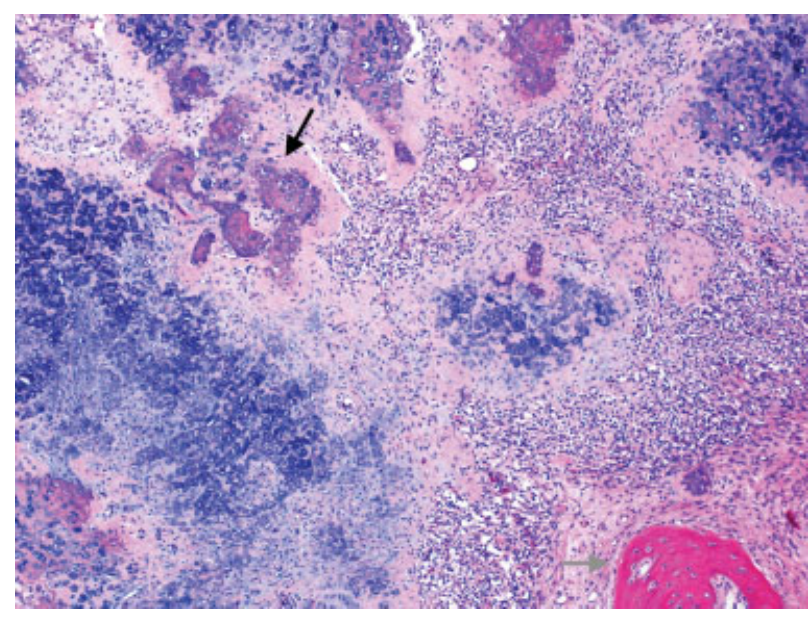

Fig. 4 Hematoxylin and eosin stain demonstrating densely cellular neoplasm with predominately spindle cell morphology, vascular proliferation, and focal mineralization. 


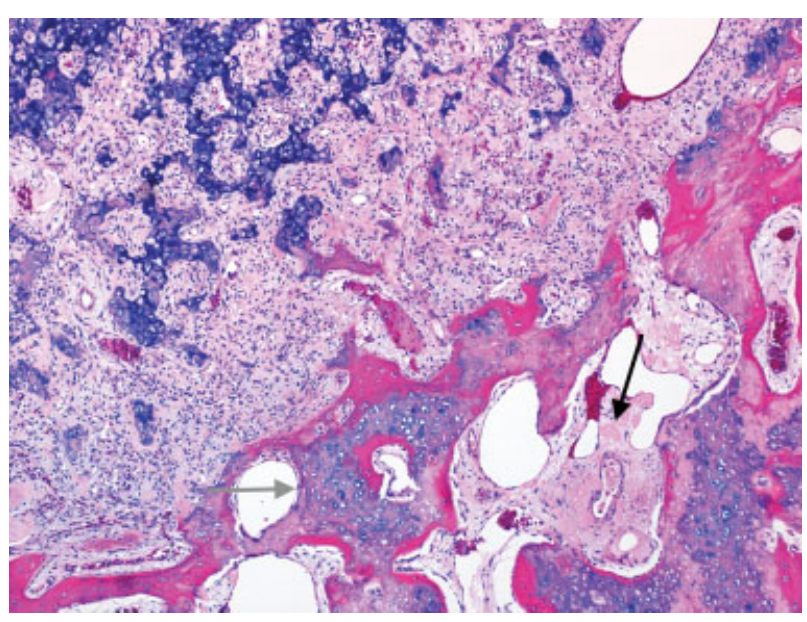

Fig. 5 High power view of hematoxylin and eosin stain demonstrating densely cellular neoplasm with high mitotic index, areas of necrosis, and regions of cartilage formation.

Differential diagnosis includes hemangiopericytoma, mesenchymal chondrosarcoma, synovial sarcoma, anaplastic meningioma, gliosarcoma, melanoma, and metastatic spindle cell carcinoma. Immunostaining is helpful to eliminate meningioma on the basis of EMA negativity, while GFAP negativity rules out astrocytic tumors. Evidence of direct osteoid formation by the tumor cells is key to the proper diagnosis. $^{3}$
Given the relative rarity of intracranial osteosarcoma, there are no established guidelines for treatment, and therapy is guided by experience with systemic osteosarcoma. Gross total resection is recommended whenever feasible. Both chemotherapy and radiation therapy are used as adjuvant therapy. ${ }^{4,5}$ Regardless of treatment, osteosarcoma remains a highly aggressive malignancy with a poor prognosis. Five-year survival is between 25 and $37 \%$. Median survival of 12 to 18 months has been reported. ${ }^{1,5}$ Morbidity and mortality may be the result of local recurrence or development of pulmonary or skeletal metastasis.

\section{References}

1 Paulus W, Scheithauer BW, Perry A. Mesenchymal, non-meningothelial tumours. In: Louis DN, Ohgaki H, Wiestler OD, et al, eds. WHO Classification of Tumours of the Central Nervous System. Lyon, France: International Agency for Research on Cancer; 2007: 173-176

2 Cihan YB, Kaplan B, Deniz K, et al. Primary calvarial osteosarcoma: a case report. J Neurol Sci (Turkish) 2012;1:122-128

3 Prayson RA. Non-glial tumors. In: Prayson RA, ed. Neuropathology. Philadelphia, PA: Elsevier; 2012:518-521

4 Ashkan K, Pollock J, D’Arrigo C, Kitchen ND. Intracranial osteosarcomas: report of four cases and review of the literature. J Neurooncol 1998;40(01):87-96

5 Setzer M, Lang J, Turowski B, Marquardt G. Primary meningeal osteosarcoma: case report and review of the literature. Neurosurgery 2002;51(02):488-492, discussion 492 